\title{
Las configurraciones didácticas, una posibilidad de la lectura crítica en el primer ciclo de primaria*
}

\author{
Maryori Berrio Ortiz \\ Institución Educativa Fe y Alegría El Limonar, Medellín,Colombia \\ may02056@hotmail.com
}

Leidy Caterine Cartagena Villegas

Institución Educativa Jesús María El Rosal, Medellín,Colombia leidycartagenav@gmail.com

Sandra Liliana Giraldo Ramírez

Institución Educativa ASIA Ignaciana, Medellín,Colombia saligira77@gmail.com

Andrés Felipe Gómez Mejía

Institución Educativa San Antonio de Prado, Medellín,Colombia felipegomez56@hotmail.com

\section{RESUMEN}

Este artículo pretende mostrar algunos resultados emanados de la investigación Prácticas de lectura crítica con estudiantes de los grados $2^{\circ}$ y $3^{\circ}$, una posibilidad de interlocución con su contexto a través de configuraciones didácticas. Se implementaron tres configuraciones didácticas como una estrategia metodológica para el fortalecimiento de la lectura crítica en el primer ciclo de la básica primaria en tres instituciones públicas de la ciudad de Medellín.

La investigación se realizó desde un enfoque cualitativo con un diseño microetnográfico escolar y la utilización de confi- guraciones didácticas como estrategia. Los resultados indicaron que la lectura crítica es un proceso posible en los primeros grados de la básica primaria, especialmente cuando el docente diseña e implementa actividades estructuradas y articuladas que responden a las necesidades e intereses de los estudiantes. De esta manera, se generan espacios en los que se les permite leer diferentes tipos de texto para debatir, preguntar, analizar y reflexionar sobre lo que ocurre a su alrededor, de manera que la lectura crítica se convierte en una posibilidad de interlocución con sus contextos.

Palabras clave: lectura crítica; configuraciones didácticas; contexto; primer ciclo.

* Cómo citar: Berrio Ortiz, M., Cartagena Villegas, L. C., Giraldo Ramírez, S. L. y Gómez Mejía, A. F. (2019). Las configuraciones didácticas, una posibilidad de la lectura crítica en el primer ciclo de primaria. Ciencias Sociales y Educación, 8(15), 105-129. DOI: https://doi.org/10.22395/csye.v8n15a6 Recibido: 15 de febrero de 2019.

Aprobado: 3 de junio de 2019. 


\section{Educational Configurations, a Possibility from the Critical Reading in the First Cycle of Primary School}

\section{ABSTRACT}

This article aims to show some results derived from the research Critical reading practices with students from the 2nd and 3rd grades, a possibility for interlocution with their context through didactic configurations. Three didactic configurations were implemented as a methodological strategy for the strengthening of critical reading in the first cycle of elementary school in three different public schools in the city of Medellin.

The researched was developed from a quantitative focus with a school multiethnographic design and the use of didactic configurations as a strategy. The results indicate that critical reading is a possible process in the first grades of primary school, especially when the teacher designs and implements activities structured and articulated that answer to the needs and interests of the students. Thus, it results in the generation of spaces for reading different kinds of texts for debate, asking, analysis and reflection about what happens in their surroundings and making critical reading a possibility for interlocution with their contexts.

Keywords: critical reading; didactic configurations; context; first cycle.

\section{As configurações didáticas, uma possibilidade da leitura crítica no primeiro ciclo do Ensino Fundamental}

\section{RESUMO}

Este artigo apresenta alguns resultados advindos da pesquisa Práticas de leitura crítica com estudantes do $2^{\circ}$ e $3^{\circ}$ ano do Ensino Fundamental, uma possibilidade de interlocução com seu contexto por meio de configurações didáticas. Foram implantadas três configurações didáticas como estratégia metodológica para fortalecer a leitura crítica no primeiro ciclo do Ensino Fundamental em três instituições públicas da referida cidade.

A pesquisa foi realizada sob uma abordagem qualitativa com um desenho microetnográfico escolar e a utilização de configurações didáticas como estratégia.
Os resultados indicaram que a leitura crítica é um processo possível nos primeiros anos do Ensino Fundamental, especialmente quando o docente elabora e implanta atividades estruturadas e articuladas que respondem às necessidades e interesses dos estudantes. Dessa maneira, são gerados espaços em que é permitido ler diferentes gêneros textuais para debater, perguntar, analisar e refletir sobre o que ocorre a seu redor, a fim de que a leitura crítica se torne uma possibilidade de interlocução com seus contextos.

Palavras-chave: leitura crítica; configurações didáticas; contexto; primeiro ciclo. 


\section{Introducción}

Para Julián De Zubiría (2015), una persona que todavía no domina la lectura crítica tiene graves limitaciones para interpretar la realidad material y simbólica. Además, afirma que la lectura y la escritura de calidad nos liberan del tiempo y del espacio en el que vivimos y nos permiten trascender, interactuar y dialogar con personas en múltiples contextos históricos, culturales y regionales. De acuerdo con el autor, los resultados de las pruebas PISA que han sido aplicadas en Colombia (2015), muestran que solo tres de cada mil jóvenes escolarizados tienen un nivel de lectura crítica a los 15 años. Por lo tanto, la carencia de esta forma de lectura no les permite distinguir matices en las afirmaciones, captar los pensamientos profundos que subyacen a los textos y encontrar posibles incoherencias en las ideas de un autor.

Ante estos datos tan contundentes, se han comenzado a explorar y a realizar propuestas pedagógicas para mejorar estos resultados a lo largo y ancho del país. Actualmente, la lectura crítica se constituye en un proceso fundamental para la educación. Por ello, se ha pretendido, desde referentes de calidad, que los estudiantes logren comprender, descubrir, analizar, reflexionar y crear discursos críticos que dejen en evidencia sus conocimientos, opiniones, sentimientos y pensamientos.

Los resultados en las Pruebas Saber en el área de lengua castellana de los estudiantes del grado tercero (2015-2016) de las Instituciones Educativas ASIA Ignaciana, Fe y Alegría el Limonar y Jesús María el Rosal, reflejaron dificultades con relación al reconocimiento de los elementos mínimos que les permita identificar la información contenida en un texto de forma literal, inferencial y crítico intertextual. Además, demostraron poca familiarización con los diferentes tipos de texto y sus propósitos comunicativos. Por ello, les costaba cuestionar al autor y expresar sus puntos de vista, además de lograr establecer relaciones con su contexto.

Los recientes estudios sobre la formación de lectores críticos como el de Murcia (2012) que se llama "Hacia la formación de lectores críticos: Un estudio de caso de clases de historia y literatura", se desarrolló en la Institución Educativa Manuela Beltrán, de San José del Guaviare en el grado noveno. Se desarrolló otro por los investigadores Álvarez-Álvarez y Pascual-Díez (2013) de la Universidad de Cantabria en España, el cual denominaron "Estudio de caso sobre la formación e lectores críticos mediante textos literarios en la educación primaria". Ambos reconocen la importancia de la formación de lectores críticos en la básica primaria. En cuanto a la utilización de otras estrategias de aula para generar la lectura crítica, se encontró el trabajo de Zuluaga (2016) que se denominó "Formación de lectoras críticas desde la literatura hacia otros 
sistemas simbólicos", en el cual se propone la reconfiguración de las prácticas de enseñanza de la literatura a partir de otros sistemas simbólicos. Por último, se encontró la investigación "La lectura crítica un encuentro entre subjetividades" realizada por Rocha (2016) en la cual se busca comprender cómo los procesos de lectura crítica desarrollados en el ámbito escolar de la Institución Educativa La Pintada abren espacio a posibilidades de tipo cognitivas, sociales y culturales.

La investigación "La lectura crítica un encuentro entre subjetividades" realizada por Rocha (2016), se entiende la lectura crítica como una lectura que permite al ser humano conocer su mundo, situarse en él y transformarlo a partir de sus vivencias. Por ello, el objetivo de esta investigación consistía en comprender cómo los procesos de lectura crítica desarrollados en el ámbito escolar de la Institución Educativa La Pintada abren espacio a posibilidades cognitivas, sociales y culturales porque este proceso permite que las personas accedan a información y construyan nuevos conocimientos a partir de sus experiencias, además de tener un rol activo en la transformación de sus contextos.

De acuerdo con las anteriores investigaciones realizadas a nivel local, regional, nacional e internacional, se puede determinar que la atención a los procesos de lectura crítica se ha centrado en los grados superiores de la primaria y de la secundaria. Estos estudios señalan que el problema radica en que los estudiantes tienen pocos hábitos lectores, sienten poco placer por la lectura y no leen de forma crítica y comprensiva. Estos aspectos les impide relacionar conocimientos, aprender con facilidad y aplicar dichos saberes en su vida diaria. En este sentido, reconocen la importancia de la lectura crítica como eje transversal en el proceso de enseñanza y aprendizaje en los diferentes entornos en los que se mueven los estudiantes. A través de la lectura crítica los estudiantes, logran interactuar con diferentes signos y símbolos que le aportan información para comprender, analizar, cuestionar, consolidar pensamientos y posicionamientos que ayuden en la transformación de su contexto y en su formación como ciudadanos.

En estos trabajos, en los cuales predominó el enfoque cualitativo de investigación, los investigadores se situaron en el campo empírico donde se dieron los hechos y, de acuerdo con la intencionalidad y el tipo de realidad que buscaban explicar, seleccionaron diferentes enfoques, como el descriptivo, los estudios de casos, la investigación acción y los diseños experimentales, los cuales tuvieron mayor aplicación. Estos enfoques permitieron observar la realidad tal y como se presentó, brindaron herramientas para que se lograra dar respuestas a los interrogantes que se plantearon para construir y aportar diferentes conocimientos.

Una vez descrita la importancia de la lectura crítica en las prácticas de 
enseñanza, se llegó a la consideración de que mediante el proyecto se podía contribuir a la formación de lectores críticos desde los primeros años de escolaridad. Así, se motivó a los educandos a participar en prácticas de lectura que fueran de su agrado e interés donde tuvieran roles importantes y se vieran involucrados, de tal forma que pusieran en práctica algunas de las habilidades propias de la lectura crítica planteadas por Medina Moya (2011), tales como descifrar el texto, contextualizar, relacionar, extrapolar informaciones y hacer una valoración del texto.

\section{La lectura crítica y algunos teóricos}

Las propuestas teóricas de Lerner (2002) en su texto "La autonomía de lector" hablan sobre la formación de lectores críticos y autónomos en el aula, dado que la escuela ha visto al estudiante como un sujeto dependiente en el proceso de lectura. En este artículo la autora establece que es necesario que el docente se apropie de diferentes estrategias didácticas que le permitan acercar a los estudiantes a ser lectores críticos y autónomos. De la misma manera, Cassany (2003) en su artículo "Aproximaciones a la lectura crítica: teoría, ejemplos y reflexiones" explora la lectura crítica a partir de aspectos teóricos y prácticos con el fin de reflexionar sobre la necesidad de fomentar este tipo de práctica lectora en el aula porque el mundo de hoy es multicultural y globalizado. Requiere de seres formados en ciudadanía que posean habilidades críticas de lectura, escritura y pensamiento para que puedan participar en la construcción y desarrollo de una sociedad más plural, respetuosa y progresista. En este mismo sentido, en el texto Explorando las necesidades actuales de comprensión de Cassany (2005),", el autor hace un rastreo sobre la lectura concebida como una práctica cambiante. Analiza esas transformaciones en el marco de una perspectiva sociocultural y expone las competencias receptivas críticas de las que debe disponer un lector crítico y los niveles de comprensión que se logran desarrollar.

De igual manera, Serrano y Madrid (2007) en su artículo "Competencias de lectura crítica. Una propuesta para la reflexión y la práctica" conciben la lectura crítica como una capacidad que debe tener la atención de la educación actual, pues contribuye a la formación de ciudadanos que reflexionen, cuestionen y tengan un pensamiento autónomo, con la capacidad de interpretar ideas, identificar puntos de vista, intencionalidades e imaginarios expresados en diversos textos que circulan en el medio.

En este orden de ideas, Jurado (2008) en su artículo "La formación de lectores críticos desde el aula" invita a los docentes a formar lectores críticos en la escuela para dejar de lado el enfoque alfabético de la lectura y trascender en la interpretación crítica de los textos. Por ello, propone varias estrategias para abordar 
este proceso, entre ellas el dialogismo intertextual que consiste en reconocer diversos textos dentro de un texto. De igual manera, señala la importancia del trabajo pedagógico a través de proyectos de aula donde los docentes en el uso pleno de su autonomía promuevan la lectura de textos literarios breves, como minicuentos, novelas y minificciones, para motivar a los estudiantes y favorecer su formación.

Desde estos planteamientos, la investigación se interesó por trabajar la lectura crítica a partir de configuraciones didácticas, dado que es la forma particular que utiliza el docente para llevar los procesos de construcción del conocimiento de acuerdo con su campo disciplinario, prácticas metodológicas y los vínculos que establece con sus estudiantes. Se realizaron tres configuraciones didácticas, una por cada institución intervenida.

Camps y Ribas (2003) establecen que la didáctica permite describir, comprender, explicar e interpretar las prácticas o situaciones de enseñanza y aprendizaje en tanto prácticas sociales, que a su vez orientan las prácticas de enseñanza. El objeto entonces de la didáctica es nutrirse de los saberes de otras disciplinas para apoyar las prácticas de enseñanza en relación con el aprendizaje. Estas prácticas están marcadas por la voluntad de enseñar y se basan en las particularidades de los diferentes contextos, las necesidades o situaciones problemáticas presentes que dan origen al proceso de enseñanza y aprendizaje.

En este sentido, se reconoce que la construcción del conocimiento tiene lugar mediante un proceso de interlocución constante con el medio social, que en esta investigación se denominará contexto próximo, aquel con el cual el sujeto interactúa diariamente: el hogar, el barrio, la escuela, todo su entorno y lo que sucede en él. Por ello, es tan importante la manera en que el docente aborda el saber en el aula dado que de ello dependerá qué tan significativo sea o no sea el aprendizaje de los estudiantes.

Así, las configuraciones didácticas se transforman en herramientas que usa el docente para ejercer su quehacer pedagógico. Litwin (1997) las define como la forma particular que el maestro utiliza para favorecer la construcción del conocimiento, pues le permite al docente llevar y diseñar prácticas de enseñanza en contexto que alimenten de significado el campo del saber. Por ello, el docente debe revisar y determinar, según la intención del aprendizaje, cuáles son las configuraciones más apropiadas que favorecen a su grupo de estudiantes, pues también existen otras configuraciones no didácticas que solo exigen por parte del maestro y el estudiante un manejo de acciones fragmentadas, carentes de intencionalidad las cuales pocas veces logran establecer una interlocución con su contexto y que solo son reconocibles en contextos institucionales. 
La lectura es un proceso que permite que el sujeto explore su mundo y otros mundos. A través de ella puede interpretarlos de acuerdo con sus conocimientos y experiencias ya vividas, volver a la historia, transformarla y crear una nueva, encontrarse consigo mismo y con otros, conocer más, cuestionarse sobre diferentes situaciones, analizar, reflexionar, construir y refutar ideas, apoyarse en otros para argumentar con fuerza discursiva sus planteamientos y proponer soluciones o alternativas frente a ciertos problemas. Por esta razón, Delgado Uriarte (2013) afirma que la lectura, además de ser una actividad lingüística, cognitiva y comunicativa, es una práctica social y cultural que debe promoverse desde el currículo. Otorgarle un carácter social a la lectura significa, como lo afirma Cassany (2007), trascender la lectura de líneas y entre líneas para avanzar a la lectura tras las líneas, lo cual implica ir más allá de lo que el texto refiere en términos de lo literal, de lo que se puede deducir a simple vista o a través de un rastreo visual e identificación de dónde está la información requerida.

El desarrollo y puesta en escena de estas habilidades permite que los estudiantes logren desentrañar el mensaje implícito de un contenido determinado, ir más allá de lo literal, analizarlo, evaluarlo para, con base en él, constituir ideas y opiniones con fundamentos claros. En otras palabras, "se apunta a que se llegue a leer entre líneas, a ver más allá de lo evidente, para poder así reinterpretar el mundo y, de paso, construir sentidos transformadores de todas las realidades abordadas" (Ministerio de Educación Nacional [MEN], 2006, p. 25). De esta manera, "la lectura crítica es el único procedimiento didáctico que fortalece el crecimiento de un yo autónomo, consciente y constructivo, con opiniones propias y con capacidad de compromiso con la comunidad" (Cassany, 2003, p. 128). Estos planteamientos están estrechamente relacionados con uno de los fines de la educación en Colombia planteados en la Ley 115 que se refiere al desarrollo de la capacidad crítica, reflexiva y analítica que fortalezca el avance científico y tecnológico nacional, orientado con prioridad al mejoramiento cultural y de la calidad de la vida de la población, a la participación en la búsqueda de alternativas de solución a los problemas, al progreso social y económico del país (MEN, 1994).

En el estudio realizado se evidenció que los docentes participantes del estudio consideran la lectura crítica como un proceso importante. Sin embargo, esta no es tenida en cuenta a la hora de hacer las planeaciones y hacer seguimiento a los procesos de los estudiantes. Además, no es un componente evaluado explícitamente en las pruebas estandarizadas para los primeros grados. Por lo tanto, este artículo presenta algunas orientaciones a través del trabajo con configuraciones didácticas en el aula para contribuir al fortalecimiento de las habilidades propias de este proceso. 


\section{La metodología}

La investigación se desarrolló bajo el paradigma cualitativo con base en el enfoque histórico hermenéutico y un diseño microetnográfico escolar, de manera que se lograra comprender cómo las configuraciones didácticas llevadas al aula permiten la formación de lectores críticos en los grados $2^{\circ}$ y $3^{\circ}$ de la básica primaria, como una posibilidad de interlocución con su contexto.

El paradigma cualitativo brindó la posibilidad de comprender las realidades de los participantes del estudio, así como las relaciones, contextos y todos los elementos que se entretejen en el proceso de enseñanza-aprendizaje. De esta manera, se logró determinar de qué forma las configuraciones didácticas llevadas al aula permiten desarrollar el proceso de formación de lectores críticos en los grados $2^{\circ}$ y $3^{\circ}$ de la básica primaria con la posibilidad de relacionarse con el contexto. Además, el enfoque histórico hermenéutico permitió a los investigadores estar inmersos en el proceso interpretativo de sus prácticas como docentes y de las experiencias de los estudiantes, con el fin de dotar de sentido y significado la información que se logró extraer de allí a la luz de los objetivos propuestos.

A través de la microetnografía escolar se tuvo la posibilidad de enfocarse en las actividades particulares del aula para construir y reconstruir una problemática no solo observada sino vivenciada, lo cual permitió buscar alternativas o propuestas que generaran cambios. Además, se utilizaron técnicas y estrategias de interacción como la observación participante, la entrevista semiestructurada, los grupos focales, los grupos de discusión y el diario de campo los cuales dejaron observar y registrar diferentes asuntos que se vinculaban al propósito de la investigación.

El proceso se desarrolló en cuatro fases. En la primera se realizaron entrevistas que permitieran conocer y caracterizar las concepciones de los maestros sobre el uso de configuraciones didácticas y la promoción de lectura crítica en el aula. En la segunda fase se diseñaron y aplicaron un conjunto de configuraciones didácticas para promover estas prácticas de lectura crítica en los grados mencionados. En la tercera fase, se analizaron cómo dichas configuraciones posibilitaron la promoción de la lectura crítica en la escuela. Finalmente, se formularon orientaciones pedagógicas y didácticas para el fortalecimiento de este proceso.

\section{Los instrumentos}

Se realizó una entrevista semiestructurada a los estudiantes de los grados segundo y tercero y a todos los docentes de básica primaria de las tres insti- 
tuciones participantes con el objetivo de recoger las diferentes percepciones acerca de cómo identificaban o reconocían el proceso de lectura e indagar sobre sus conocimientos en el uso de las configuraciones didácticas y la incidencia que estas tenían al momento de mediar los diferentes procesos de lectura con los estudiantes, especialmente en los que hace referencia a la lectura crítica. También se realizaron entrevistas a estudiantes frente sus gustos por la lectura, sus concepciones sobre la importancia de esta en relación con su contexto cercano y su interpretación.

La información obtenida en estas entrevistas fue registrada en matrices de análisis. Los resultados constituyeron el insumo para el diseño de las tres configuraciones didácticas. Las diferentes percepciones y apreciaciones, en el caso de los docentes, mostraron la necesidad de estructurar una serie de actividades que estuvieran intencionadas y articuladas con el currículo en la formación de lectores críticos, ya que algunos de éstos expresaron que hacían uso de estrategias desligadas entre sí y descontextualizadas en sus prácticas de aula. En el caso de los estudiantes, la expresión de sus gustos por la lectura mostró la importancia de generar situaciones que no solo les permitiera hacer lecturas de manera literal, sino también de manera crítica en relación con su contexto porque su cotidianidad los marca.

Otro instrumento utilizado en la investigación para el registro de lo que acontecía durante el desarrollo de las configuraciones fue el diario de campo, el cual fue complementado con el diseño de tres rúbricas que fueron aplicadas al finalizar cada sesión. La primera de ellas contenía aspectos propios de las competencias de un lector crítico: competencias cognitivas para la identificación de puntos de vista intencionalidades; competencias lingüísticas y discursivas para reconocer el género discursivo (estructura, registro, funciones y recursos lingüísticos); competencias pragmáticas y culturales para derivar implicaciones y cuestionar cómo se posicionan las ideas; competencias valorativas y afectivas para reconocer contenidos, opiniones, presupuestos y valores estéticos. Se evaluaron con rendimiento Alto, Medio y Bajo.

La segunda rúbrica se realizó para evaluar si la configuración había logrado motivar al estudiante para descifrar su contexto cercano, relacionar la información de los textos leídos con su contexto, valorar la información que el texto le proporcionó para comprender su contexto cercano, ampliar sus ideas y realizar conclusiones sobre la temática abordada, mostrar interés por comprender algunos sentidos implícitos en el texto. Estos desempeños se valoraron con Sí y No y se realizaron observaciones.

La tercera rúbrica tenía como finalidad registrar las opiniones de los estudiantes sobre las actividades realizadas, con preguntas tales como: ¿Qué te 
pareció la actividad? ¿Por qué? ¿Qué aprendiste de nuevo? ¿Para qué te sirve lo que aprendiste? ¿Qué momento te gustó más y por qué? ¿Conocías algo de lo que se trabajó en la actividad? ¿Qué conocías? Si pudieras cambiar algo de las actividades, ¿qué cambiarías y por qué? ¿Qué otro tema te gustaría abordar? La valoración de esta rúbrica fue abierta.

\section{Los participantes}

El estudio se realizó en tres Instituciones Educativas de carácter público del municipio de Medellín: ASIA Ignaciana, Fe y Alegría el Limonar y Jesús María el Rosal. Cada una tiene unas características sociales y culturales distintas.

La población que participó del estudio estuvo conformada por 120 estudiantes con edades entre los siete y diez años. Sin embargo, los análisis investigativos se hicieron a un grupo de 30 estudiantes de las tres Instituciones, quienes fueron distribuidos de la siguiente manera: diez estudiantes de tercero y veinte estudiantes de segundo.

\section{El trabajo de campo}

Se diseñaron tres configuraciones didácticas, una para cada institución: 1) Los proyectos de aula en Institución Educativa Jesús María el Rosal, 2) las secuencias didácticas en la Institución Educativa ASIA Ignaciana y 3) la enseñanza para la comprensión en la Institución Educativa Fe Y Alegría Limonar.

Las configuraciones se enmarcaron en temáticas que formaban parte de la cotidianidad de los contextos de los estudiantes de las tres instituciones, entre ellas el cuidado del cuerpo, la afrocolombianidad, la paz, el desplazamiento y el entorno protector. Cada docente tenía la autonomía para gestionar las actividades y disponer de diversas herramientas y portadores de textos. La selección de estas temáticas se realizó con la intención de promover prácticas de lectura crítica que contribuyeran al proceso de aprendizaje de los estudiantes y los llevara a interpretar, comparar y proponer diferentes estrategias ante su realidad social o contexto cercano, además de adquirir un sentido social que va más allá de la simple decodificación de palabras y frases.

\section{Los resultados}

Una vez concluida la implementación de las configuraciones, se procedió a realizar la interpretación y análisis de los resultados a la luz de las postulaciones teóricas sobre la lectura crítica, las configuraciones didácticas y la interlocución con el contexto, además de las otras subcategorías que emergieron durante la investigación como la evaluación, tipologías textuales y prácticas pedagógicas. 
Estas brindaron sustento para dar cumplimiento al último de los objetivos y fases de la investigación que consistía en proponer un conjunto de orientaciones pedagógicas y didácticas para los docentes de la básica primaria de las tres instituciones educativas.

\section{Los referentes de calidad y la lectura crítica}

Se hizo una revisión documental a los referentes de calidad con el fin de conocer y analizar las orientaciones por parte del Ministerio Nacional de Educación en torno a la lectura crítica. En los Lineamientos Curriculares de Lengua Castellana (1998) se encontró que existe un llamado a abordar la lectura desde la formación de lectores críticos a partir de los primeros grados basada en sus competencias. En este documento se plantea que la lectura crítica es uno de los niveles en los que se pueden ubicar los estudiantes de acuerdo con su proceso y las habilidades que desarrollan en los diferentes grados escolares a la hora de leer. Por otro lado, en los Estándares Básicos en Competencias del Lenguaje (2006) no se hace una referencia directa y explícita de la lectura crítica. En los Derechos Básicos de Aprendizaje DBA (2016), que es el referente de calidad más actual que orienta los currículos escolares, solo se hace una pequeña mención del concepto como acción de comprender determinado contenido a partir de la estructura en la que está construido.

\section{Lo que muestran las pruebas estandarizadas sobre la lectura crítica}

Los resultados de las pruebas PISA a nivel a nacional vienen indicando que estamos haciendo las cosas mal, así lo confirma De Zubiría (2015) cuando afirma que en Colombia solo tres de cada mil jóvenes escolarizados tienen un nivel de lectura crítica a los 15 años. El autor señala que esta habilidad les permite distinguir matices en las afirmaciones, captar los pensamientos profundos que subyacen a los textos y encontrar posibles incoherencias en las ideas de un autor. Asimismo, hace referencia a la construcción de ciudadanía, considera a la lectura comprensiva como condición de la democracia. Y se hace las siguientes preguntas:

¿Cómo serán las dificultades que tienen estos mismos jóvenes para distinguir matices en las ideologías de los partidos políticos? ¿Cómo serán las limitaciones que tienen para comprender la conveniencia de un programa político, económico o cultural y para evidenciar lo equivocado que pueda ser elegir a un candidato en unas elecciones locales o regionales?

Mientras los niveles de comprensión lectora en el país permanezcan así no podremos hablar de democracia y libertad. Necesitamos ciudadanos que puedan elegir y construir su propio destino. 
El país acaba de pasar por una jornada electoral que definió los gobernantes locales y regionales para los próximos cuatro años. La conclusión es muy clara: mientras no mejoremos de manera sensible la calidad de la educación no podremos hablar de que vivimos en una democracia. Los niveles ínfimos de lectura crítica que hoy alcanza nuestra población demuestran que no estamos en la capacidad de elegir adecuadamente.

En Colombia, millones de personas tienen más de dieciocho años, pero muy pocos son mayores de edad en el sentido kantiano; es decir, muy pocos tienen herramientas y criterio para pensar por sí mismos. Sin lectura crítica no es posible elegir de manera responsable. Es por ello, que mejorar la calidad de la educación es una condición sine qua non para que podamos decir que vivimos en una democracia. (parr. 5)

Los anteriores planteamientos señalan la necesidad de promover estrategias para desarrollar la lectura crítica en los estudiantes desde los primeros grados escolares. Esta necesidad se evidenció en la revisión de los resultados de las Pruebas Saber del área del lenguaje de los estudiantes del grado tercero (20152016) pertenecientes a las tres instituciones públicas participantes del estudio, donde se encontró que pocas veces lograban dar cuenta de la información literal del texto, hacer inferencias y hacer una lectura crítica e intertextual. Además, mostraron poca familiarización con los diferentes tipos de texto y sus propósitos comunicativos.

\section{¿Qué nociones tienen los docentes y estudiantes sobre la lectura crítica?}

En las entrevistas a docentes y estudiantes se encontró que los docentes le ven un fin lúdico a la lectura como herramienta motivadora, que divierte a los estudiantes y facilita sus aprendizajes. Sin embargo, no se refieren a la lectura como posibilidad de pensamiento, de reflexión y construcción de un conocimiento crítico. Los estudiantes, por su lado, visualizan la lectura como un mero mecanismo de aprendizaje que es evaluado con preguntas abiertas o cerradas que solo sirven para medir y cuantificar saberes sobre un tema determinado o una comprensión literal de lo que leen.

De acuerdo con las respuestas de los docentes sobre la lectura crítica, se evidenció que estos reconocen los beneficios de este tipo de lectura. Sin embargo, consideran que esta requiere procesos elaborados y estructurados de un mayor nivel que el que poseen los estudiantes de $2^{\circ}$ y $3^{\circ}$. Esta idea limita el proceso de los niños, los restringe de vivenciar el poder de la lectura como una manera de habitar y comprender el mundo tal. Asimismo, se pudo constatar que los docentes como sujetos partícipes en el proceso educativo, tratan de cumplir con los contenidos temáticos establecidos en el currículo. Para ello, recurren a diversas estrategias didácticas y pedagógicas que incluyen la lectura como una 
acción a partir de la cual los estudiantes pueden extraer información, comprender y analizar. Pero se limitan al uso del texto narrativo, donde la comprensión es evaluada, en la mayoría de los casos, con preguntas que se circunscriben en el nivel literal.

Los estudiantes, por su lado, expresaron que leían tanto en la casa como en la escuela diversas tipologías textuales por placer, mientras que en la escuela leían por requerimientos académicos y objetivos planteados previamente por el docente. También expresaron que debían dar cuenta de lo leído con talleres que, en vez de motivarlos, los aburrían y los abrumaban, pues no poseían la capacidad para la decodificación y la comprensión de los textos. A pesar de que los estudiantes no viven a menudo experiencias que los motiven a leer en la escuela, muchos manifestaron tener gusto por la lectura y ser conscientes de que esta es importante porque les permite acceder a nuevos conocimientos, a divertirse, a aprender y a enriquecer su vocabulario.

\section{Algunas competencias para ser un lector crítico}

Aportar a la formación de lectores críticos desde la escuela es una necesidad apremiante para una sociedad que exige sujetos con capacidades que le permitan interpretar su contexto en la búsqueda de una transformación social. Por ello es necesario comprender que,

desde la escuela se pueden formar lectores críticos y no solo lectores alfabetizados. Esta distinción es de gran importancia: una cosa es educar en la escuela para alfabetizar y otra orientar desde la escuela para saber leer críticamente los textos de la cultura. (Jurado, 2008, p. 90)

En cada una de las configuraciones implementadas se buscó que los estudiantes lograran apropiarse de algunas competencias necesarias para la lectura crítica, tales como la capacidad para descifrar el texto, contextualizarlo, relacionarlo, extrapolar las informaciones y hacer una valoración del texto. La competencia cognitiva se evidenció a través de algunas intervenciones de los estudiantes, en la forma cómo hacían uso del lenguaje oral y escrito a partir de saberes previos y esquemas de pensamiento estrategias para interpretar, hacer inferencias y dar cuenta de lo que sabían sobre la información presente en los textos. Esta afirmación se puede corroborar en este fragmento: "A la hija de una señora por mi casa el tío la tocó" (Estudiante anónimo, comunicación personal, 30 de julio de 2018).

Al leer las respuestas de los estudiantes, se encontró que algunos de ellos seleccionaron con propiedad varias situaciones presentes en el texto y las contrastaron con su contexto. De esta manera, lograron desentrañar y apropiarse 
de parte del discurso dando cuenta de éste en forma oral, fortaleciendo las competencias lingüísticas y discursivas, pues al conocer o tener referencias de los casos presentes en su contexto contados por sus compañeros de clase, les era más cómodo explicar de forma oral la situación y comprender posibles variables y situaciones futuras. En algunas de sus respuestas se pudo apreciar que reconocían la intencionalidad de lo leído, se apoyaban en las acciones de los personajes para comparar y hacer reflexiones con sus experiencias más cercanas, lo que se evidencia en el siguiente fragmento: "Es bueno lavarse las manos, para evitar maluqueras, y comer bien y estar hidratado todo el tiempo, para poder responder a las cosas de la escuela y en el infograma están y nos enseña muchas cosas buenas sobre la salud" (Estudiante anónimo, comunicación personal, 3 de agosto de 2018).

Estas respuestas evidencian la manera en que los niños dejan ver que han comprendido parte de la información, pues hacían una reflexión frente a la importancia que esta tiene en relación con la salud, no solo para ellos, si no de todas las personas que leen el texto. Además, le daban validez a las respuestas del portador de texto utilizado por el docente, dado que en el grado $2^{\circ}$ el estudiante debe estar en la capacidad de leer y explicar el mensaje principal de un texto escrito o un gráfico y el del grado $3^{\circ}$ debe estar en la capacidad de realizar inferencias y relaciones coherentes. Por otro lado, también se tiene en cuenta la capacidad de realizar intervenciones orales sobre el tema tratado, una lectura o un evento significativo. Esto muestra que cuando la escuela o el docente propician momentos de lectura y permiten que los estudiantes profundicen, comienzan a apropiarse de las competencias necesarias para ser un lector crítico. De ahí la importancia de que el docente identifique con claridad estas competencias y abandone la idea de que leer, en los primeros grados, consiste en el simple hecho de decodificar que se centra solo en un nivel literal.

Las competencias pragmáticas o comunicativas que se refieren a la competencia funcional o a la intencionalidad del hablante y las competencias culturales que son las de apropiación del patrimonio lingüístico, tienen lugar en los hallazgos cuando los estudiantes dejaban ver en sus respuestas que poseían la habilidad de usar palabras, dichos y términos del contexto. Esto quiere decir que utilizaban lo que podría llamarse el parlache para crear nuevas y resignificadas propuestas expresivas a partir del uso de sus ideas y representaciones de los diversos entornos culturales y sociales. Es así como un lector crítico se vale del texto para reconstruir su pensamiento, dotarlo de nuevos significados, comparar los planteamientos de dicho texto con otros y encontrar intencionalidades e ideas implícitas en los discursos. Lo anterior solo tiene lugar cuando hay un proceso de comprensión previo que conlleva a la interpretación y, finalmente, a la lectura crítica, como se puede evidenciar en la respuesta de un estudiante 
a un docente en torno a la pregunta sobre cómo se puede vivir mejor según el cuento. En este sentido, el estudiante responde: "Él nos quiere enseñar a cuidarnos de los pillos, él nos enseña a defendernos de los combos, que uno debe hablar siempre para no tener problemas" (Estudiante anónimo, comunicación personal, 25 de septiembre de 2018).

De igual manera, se evidencia el uso de competencias valorativas y afectivas, pues la lectura permitió entender que no todos los sujetos les deseaban hacer daño y que existen muchas personas que los quieren y los respetan. Las respuestas, las cuales son coherentes con lo que planteaba el texto, estaban cargadas de la subjetividad propia de quien habla y su interpretación personal del contexto, lo cual se ratifica en estos fragmentos: "Profesora yo creo que las personas que cuidaron al niño del cuento son héroes de la vida real, porque ayudaron al niño" (Estudiante anónimo, comunicación personal, 13 de agosto de 2018). Otro estudiante respondió lo siguiente a la pregunta del docente sobre la importancia de alimentarnos de manera saludable: "En el colegio las niñas comen más papitas y jugos de cajita y eso no es saludable" (Estudiante anónimo, comunicación personal, 25 de julio de 2018).

Las experiencias de lectura mediadas por las configuraciones didácticas contribuyeron a la apropiación de algunas de las competencias de un lector crítico, tales como las cognitivas, las lingüísticas y discursivas, las pragmáticas y culturales, las valorativas y afectivas, que permitieron una interlocución significativa con el contexto. En este sentido, se logró dar cuenta de las intencionalidades y los mínimos básicos para los cursos, establecer que los niños identificaron las palabras en familias y en las actividades del barrio o de la cuadra. A su vez, leyeron, explicaron los mensajes de los textos y de los gráficos y los relacionaron con sus conocimientos experiencias, así como también vieron las consecuencias de dichas acciones y mensajes. Esto demuestra que realizaron inferencias, relaciones coherentes y, especialmente, realizaron intervenciones orales sobre los temas tratados y las explicaron con sus propias palabras en relación con sus entornos.

\section{Configuraciones didácticas: desde el pensar y el hacer}

El trabajo por proyectos de aula posibilitó la participación de los estudiantes porque su diseño parte de temáticas de interés con el fin de generar experiencias significativas e integrar saberes que giran en torno a la realidad, las necesidades y las experiencias de los estudiantes. En esta tarea, el docente llevó al aula procesos que les permitieran investigar y aprendieran a desarrollar la autonomía mediante la elaboración de ideas en común. Crearon y formularon sus propios puntos de vista como una de las habilidades propias de la lectura 
crítica, yendo de la simple apropiación de significados a la argumentación de sus propios puntos de vista, como se puede observar en el diario de campo cuando una docente decía: "Con esta configuración didáctica se pudo acercar los niños y niñas a la realidad que vive el país utilizando diferentes formatos, canciones, videos e imágenes" (Leidy, comunicación personal, 10 de agosto de 2018).

Las secuencias didácticas no se alejan de lo anterior. Durante la puesta en marcha de la investigación se encontró la posibilidad de trabajar en el aula estructurando y generando la posibilidad de una participación de los estudiantes, pues esto favorecía la posición argumentada de ideas y puntos de vista. La secuencia didáctica partió de necesidades reales del contexto que, llevadas al aula de manera intencional, se articularon bajo una idea central que enlazan diferentes saberes. En este punto, el trabajo del docente consistió en determinar esas acciones y priorizarlas, de manera que los propósitos de aprendizaje pudieran alcanzarse.

En cuanto a la enseñanza para la comprensión, se encontró que en las planeaciones de los maestros entrevistados esta configuración didáctica no hace parte de las prácticas pedagógicas porque se desconocen. Esta metodología, relativamente nueva, pertenece a las llamadas metodologías activas que basan su trabajo en el estudiante, quien construye nuevos conocimientos y los utiliza en situaciones reales. En este sentido, el estudiante es protagonista de su propio aprendizaje y las actividades le exigen "pensar y actuar flexiblemente con lo que saben... yendo más allá de la memoria, la acción y el pensamiento rutinario" (Perkins, 1999, p. 78). De esta manera, se reta a los estudiantes a que aprendan de acuerdo con las necesidades de su contexto, sus habilidades y saberes.

\section{Posibilidades de la lectura crítica y la interlocución con el contexto}

Es evidente que los estudiantes retoman elementos de lo que leen y los dotan de sentido a partir de lo que conocen y viven en su entorno, es decir, pasan del nivel de comprensión a una "interpretación crítica del ámbito social en el que habita el individuo" (Cardona y Londoño, 2017, p. 378). En una de las configuraciones se hizo, por ejemplo, la lectura de una noticia que tenía que ver con la mala alimentación de los niños de Medellín. Algunos estudiantes se podían identificar con este texto, así que la lectura posibilitó la constitución del vínculo entre las palabras con lo que vivían en sus casas o en la escuela en relación con la forma de alimentación.

En este sentido, "la lectura debe ser una práctica que tiene como base la aprehensión y comprensión del contexto, que es donde, finalmente, recae la acción transformadora del individuo" (Cardona. y Londoño, 2017, p. 378). Se habló de transformación porque cuando el lector comprendía la información del texto 
y la confrontaba con su vida, convertía la lectura en una práctica social que no podía estar apartada del mundo circundante. Al contrario, se encontraba ligada a él, pues al mediodía recibiría el almuerzo escolar. La realidad es inspiración para los que escriben y el medio de comprensión para los que leen.

La lectura crítica también permitió la apropiación del contexto que, a su vez, posibilitó que algunos estudiantes comentaran lo que les gustaba y lo que no les agradaba de la comida que recibían, tomando posición y expresando sus ideas en función de lo que conocían y estaban viviendo. Al respecto, Luke (2004) plantea que ser crítico constituye la formación de una autoconciencia que tiene repercusiones en la visibilización y viabilidad de los procesos sociales. Solo de esta manera el lector se convierte en un agente de transformación de su entorno que está caracterizado por la violencia, las bandas, los desplazamientos, las pocas oportunidades laborales y económicas de sus moradores, así como las llamadas fronteras invisibles y la presencia de diversidades culturales, pero especialmente las problemáticas con los territorios y su control.

\section{La pregunta y su papel en las configuraciones didácticas}

Uno de los hallazgos comunes durante la aplicación de las tres configuraciones didácticas fue que el uso de la pregunta generó en los estudiantes procesos de análisis, reflexión y argumentación, propios de la lectura crítica. Sus respuestas evidenciaron conocimientos, opiniones, sentimientos y pensamientos, lo que no solo les permitía un empoderamiento de un texto o contextos, sino que se apropiaron de los usos lingüísticos particulares de cada zona de vivienda y de las realidades presentes en sus propias casas, tal y como se evidencia en el siguiente fragmento después de leer muy fuerte el texto Estela Grita. Ante la pregunta ¿por qué grita Estela? Los estudiantes respondieron:

Por el trato de su tío, de su mamá y su mejor amiga, [...] ella no grita, pues tiene miedo de hacerlo, solo confía en su mamita, [...] ella debería de hablar y no quedarse callada, por miedo a esos manes [...] los niños tenemos que hablar con nuestros papás sobre, lo que nos pasa, y si ellos no nos escuchan con nuestros profesores. (Estudiantes anónimos, comunicación personal, 1 de agosto de 2018).

Estas respuestas evidencian la manera en que la pregunta en el aula se convierte en un recurso que sirve al docente para potenciar procesos de lectura crítica e, incluso, como potenciadora catártico para que los chicos expresen sus pensamientos. En este sentido, Zuleta (2005) plantea que el uso reflexivo de la pregunta se convierte en uno de los fundamentos del aprendizaje de un estudiante, ya que le permite gestionar sus conocimientos. De esta manera, la pregunta se consolida en una herramienta en el proceso de enseñanza y aprendizaje, siempre y cuando sea una pregunta con sentido y con un objetivo claro, ya que "[e]l sentido de preguntar consiste precisamente en dejar al descubierto la posibilidad de discutir sobre el sentido de lo que se pregunta. Una 
pregunta sin horizonte o sin sentido, es una pregunta en vacío que no lleva a ninguna parte" (Zuleta, O., 2005, p. 116). Se precisa de un sujeto crítico que no solamente asuma un rol pasivo de recibir información, sino que se cuestione. En este contexto, Freire (1986) afirma: "me parece importante observar cómo hay una relación, indudable entre asombro y pregunta, riesgo y existencia. Radicalmente, la existencia humana implica asombro, pregunta y riesgo" (p. 6).

En las respuestas de los estudiantes, a partir de los diálogos generados con el docente y compañeros, se pudo evidenciar que están en capacidad de elaborar y resolver preguntas antes, durante y después de las lecturas. Así, es posible avanzar en los procesos de lectura crítica, puesto que no solo hacen alusión al contenido textual o literal, sino que establecen una relación con su vida cotidiana, hacen análisis en situaciones puntuales y se remiten a lo que conocen por referencias extraídas de otros medios, textos o vivencias.

Muchos de los estudiantes presentaban realidades familiares y vivenciales no evidenciadas en la escuela, puesto que algunas veces ella se llega a la escuela para convertirla en ese paraíso posible donde hay comida, amigos y juego para mitigar lo que ocurre en sus contextos y en sus hogares. Los conflictos en las distintas comunas de la ciudad de Medellín muchas veces son invisibilizados por la administración municipal que, al buscar inversionistas, deja de lado las situaciones difíciles de estos contextos en los que viven muchas personas. Otras veces son los padres quienes construyen una burbuja para que sus hijos, como forma de sobrevivencia, no se den cuenta de lo que realmente sucede. Por ello, muchos de ellos quieren contar y narrar lo que les sucede, y lo que piensan de ello. A menudo lo hacen con el uso de la tercera persona.

\section{Los materiales de lectura en la formación de ciudadanos críticos}

En las configuraciones didácticas se abordaron varios tipos de texto que ayudaron en el fortalecimiento de la apropiación de algunas de las competencias de la lectura crítica. Con respecto a esto se encontró que los textos informativos, en especial la noticia, facilitaba a los estudiantes comprender y establecer relaciones con su contexto. Es una característica cultural que los comentarios del acontecer nacional sean realizados por un buen número de adultos y jóvenes en la presencia de los niños, por eso la mayoría están al tanto de lo que sucede y de alguna manera se apropian y repiten los enunciados y posturas políticas de sus padres. Los padres, a su vez, repiten las posturas de los elegidos de su agrado. Lo mismo sucede con los equipos de fútbol y con los gustos musicales. Un ejemplo de ello se puede ver en el siguiente apartado:

Más tarde, se dio a cada una de las niñas una ficha con la noticia llamada "Obesidad en niños de Medellín" y se hizo lectura en voz alta de la misma; luego realizamos un círculo de la palabra con preguntas orientadoras. En este conversatorio 
las niñas mostraron haber comprendido la noticia en los diferentes niveles de lectura dado que lograban dar cuenta de datos literales, hicieron inferencias y asumieron una postura frente a cómo debe ser la alimentación de los niños en Medellín. (Leidy Cartagena Villegas, comunicación personal, 3 de agosto de 2018).

Lo anterior muestra que las noticias que se seleccionaron de acuerdo con los sondeos en el grupo contribuyeron a descubrir la importancia que para ellos pueda llegar a tener el tema, como fue el caso de la obesidad. Este tema de las dietas y el trabajo sobre el cuerpo ocupa un lugar preponderante en sus comunidades, pero en este caso la noticia fue elegida por la renovación del menú en el Programa de Alimentación Escolar PAE y la incorporación de alimentos funcionales en los comedores educativos. Estas noticias favorecieron el proceso de lectura crítica en los estudiantes porque les permitió conocer un nuevo vocabulario, comprender cómo se articulaba la estructura del discurso expositivo, interpretar la información que había en dicho discurso y cómo esta influía en su contexto, de manera que no solo leían para buscar información, sino para analizarla y expresar sus opiniones e ideas.

\section{El trabajo colaborativo}

La lectura compartida, algunas veces en voz alta orientada por el maestro y otras por los estudiantes, fue una estrategia empleada por los docentes en el desarrollo de las diferentes actividades propuestas en cada una de las configuraciones didácticas. En el grado segundo se hizo mayor énfasis en la lectura compartida y en el grado $3^{\circ}$ en la lectura autónoma en equipos colaborativos porque dentro de los Derechos Básicos de Aprendizaje para cada grado existe una mayor complejidad a medida que es más avanzado. Pero también se pensó en una estrategia de compañerismo y solidaridad entre los niños del grado $2^{\circ}$. El objetivo en ambos grados consistió en lograr una mejor comprensión del mensaje o información contenida en determinado portador de texto y la participación activa de los estudiantes, con el fin de apropiar algunas de las competencias para la lectura crítica, muestra de ello se puede ver a continuación: El cuento fue presentado a los y las estudiantes en formato digital, la docente leía la historia mientras realizaba preguntas que les permitieran hacer anticipaciones y predicciones a los estudiantes. (Sandra Giraldo Ramírez, comunicación personal, 13 de agosto de 2018).

Las configuraciones didácticas fomentaron el trabajo colaborativo como una posibilidad para desarrollar diferentes habilidades que permitieron estar en contacto con el otro escucharlo y reconocerlo con sus capacidades a través de la distribución de roles. Crearon vínculos y establecieron pautas de trabajo planificadas para consolidar espacios de diálogo donde se compartieron saberes. Como se afirmó anteriormente, cuando alguno tenía dificultad en leer, ellos 
mismos se corregían y se explicaban la manera correcta de hacerlo, según lo expresado por el docente.

Dice Zañartu:

el aprendizaje colaborativo está centrado básicamente en el diálogo, la negociación, en la palabra, en el aprender por explicación, [...], aprender es por naturaleza un fenómeno social, en el cual la adquisición del nuevo conocimiento es el resultado de la interacción de las personas que participan en un diálogo. (Zañartu, 2003, p. 2)

De esta manera, el trabajo colaborativo se implementó mediante trabajos en parejas con la lectura en conjunto, la recomendación al otro cada vez que se equivocara el lector, la corrección hasta lograr la pronunciación correcta, la comprensión de lo expresado en el texto y si no lo podían resolver entre los dos, llamar y solicitar la ayuda del docente. Durante el desarrollo de las configuraciones didácticas se evidenció que este tipo de estrategias metodológicas permitieron consolidar objetivos en común que potenciaron la capacidad para encontrar, analizar y contrastar información de los diferentes formatos que se presentan en el aula. En el siguiente fragmento, la docente argumenta:

\footnotetext{
Esta actividad fue bastante enriquecedora porque cada equipo se pudo acercar a un testimonio de una víctima y tener la oportunidad de ponerse en su lugar, formular preguntas sobre la información que no estaba clara en cada caso o que ni siquiera era expuesta y para dar respuesta de ellas plantear hipótesis. Esto se puede ver en el siguiente apartado: ¿Por qué los otros creyeron que el esposo de Carmen era un guerrillero? Porque volvió a la mina a trabajar. (Leidy Cartagena Villegas, comunicación personal, 10 de agosto de 2018).
}

Dentro de las bondades del trabajo colaborativo y la lectura con sentido crítico, se evidenció que cada estudiante exponía la mirada y las ideas que tenía sobre el tema de acuerdo con las temáticas tratadas. También les permitió conocer las realidades de sus compañeros que eran diferentes en forma y contenido, aunque podrían compartir el mismo territorio. Se encontró de manera puntual que este estilo de trabajo favorecía la participación de aquellos estudiantes que mostraban inseguridad, ya que iban encontrando sus fortalezas en el proceso, se empoderaban y participaban. Asimismo, esta propuesta llevó a afianzar y transversalizar las temáticas de la ética de la comunicación, ya que se establecieron dinámicas donde el respeto por la opinión del otro y el punto de vista del otro eran importantes. La escucha y la participación por turnos con roles establecidos contribuyó al fortalecimiento de los vínculos grupales.

\section{Conclusiones}

Las configuraciones didácticas llevadas al aula permitieron promover la lectura crítica como una posibilidad de interlocución con el contexto. Estas implicaron el 
uso de diferentes metodologías de aprendizaje donde el docente abrió espacios para la discusión y la reflexión argumentada a través de preguntas, el trabajo colaborativo y la implementación de diferentes formatos para presentar contenidos de lectura crítica para su posible apropiación como una manera real de reconocerse y complejizarse en el contexto que se habita.

En el caso de esta investigación las tres configuraciones empleadas permitieron la promoción de las prácticas de lectura crítica, ya que poseen características didácticas que posibilitan la participación de docentes y estudiantes como sujetos del acto educativo que están en constante formación, además de que permitió la articulación de áreas y el abordaje de temáticas de interés por medio de diferentes actividades. Desde lo didáctico, la reconfiguración de espacios en el aula y la escuela, así como el contraste con las situaciones reales que acontecen en sus casas potenciaron la comprensión de sus realidades. Se aportó, así, a la construcción de ciudadanía, pues a partir de las lecturas y las expresiones orales se buscaron alternativas para mejorar las situaciones actuales cuando se les preguntaba, por ejemplo, por lo que harían en un caso específico. A partir de la situación problema de las lecturas planteadas se les explicaba cómo sus realidades no eran exclusivas a sus espacios, sino que obedecían a circunstancias de orden distintas a las escuchadas en sus hogares y vecinos. Se comenzó a complejizar de manera oral la información que el estudiante recibía. Es bueno aclarar que es la televisión y no la lectura la herramienta formativa en estos contextos socioculturales.

El trabajo a través de las configuraciones didácticas como son los proyectos de aula, las secuencias didácticas y la enseñanza para la comprensión permitió diseñar prácticas de enseñanza en contexto y transversalizar áreas, pues todos los participantes de la investigación son monodocentes. Esto quiere decir que se encargan de impartir todas las áreas al mismo grupo y articulan los procesos por medio de temas que incluyen varias áreas.

Es necesario que los docentes piensen en su quehacer docente, reflexionen y asuman el reto de transformar sus prácticas, de manera que el aula se convierta, no en un espacio de trasmisión, sino de construcción de saberes, de diálogo, de análisis y de reflexión. En este sentido, señala Carantón (2018), citando La educación en la encrucijada de Fernández Enguita (2016), que nos hemos dedicado a observar los árboles y no el bosque. De allí se desprende que hayamos atado el debate y las prácticas a lo políticamente correcto y a las retóricas de los grupos de interés, que, como todos sabemos, son demasiado estrechos y no son precisamente los educativos. Por ello, las configuraciones didácticas se convierten en uno de los caminos para lograr la apropiación de algunas de las competencias de un lector crítico, pues se tornan herramientas para mirar de forma crítica las condiciones y contextos de la sociedad. 
En cuanto a la lectura crítica, también es necesario que los maestros de los primeros grados de la básica primaria especialmente los del primer ciclo, los grados $1^{\circ}, 2^{\circ}$, y $3^{\circ}$, comprendan que esta debe ser llevada al aula de manera intencional, dado que se tiene la idea errónea de que a tan temprana edad no es necesario trabajarla. Pero como es un proceso, si aparece antes se lograría mejorar la calidad de lectura en los niveles superiores.

En esta investigación se evidenció la necesidad de incentivar y promover el trabajo tanto en el aula como en el hogar, pues se hizo fundamental la recolección de términos de uso cotidiano en la comunidad, un parlache cuyos significados difieren en los usos de acuerdo con los lugares de la ciudad donde se aplican. Estas actividades estuvieron articuladas con temas de interés que fueron relacionados con el contexto del estudiante, de manera que pudieran transversalizar saberes y comenzar a desarrollar competencias lingüísticas, discursivas, pragmáticas, culturales, cognitivas, valorativas y estéticas. En consecuencia, los estudiantes pudieron establecer relaciones dialógicas entre lo que conocían, lo que leían y lo que vivían para constituirse a futuro en un sujeto crítico que le apueste a la transformación de su entorno.

Se encontró que los estudiantes comenzaron a movilizar su pensamiento crítico, no solo cuando respondían a una pregunta intencionada del docente, sino también cuando ellos formulaban preguntas o se cuestionaban frente a lo que leían. Así, establecieron su punto de vista y lo argumentaron delante de sus compañeros, de manera que se fueron construyendo espacios para el diálogo y la participación. También se halló que los niños mostraban mayor interés cuando había un tema nuclear que articulaba las actividades con varias áreas.

El docente en su proceso de enseñanza tiene la responsabilidad de elegir materiales de acuerdo con las necesidades de los estudiantes, el contexto y los propósitos que tenga en cada una de las actividades o estrategias que lleva al aula. Uno de esos materiales en torno a la enseñanza de la lectura y la escritura tiene que ver con el tipo de texto que se va a abordar. En el caso de la formación de lectores críticos, se reconoce que cada tipología textual posee características según la forma del mensaje y su intención comunicativa las cuales favorecen procesos de lectura crítica. Sin embargo, el texto informativo de manera específica, la noticia, presenta diversas bondades dado que como discurso que busca informar situaciones del contexto, facilita que los estudiantes desarrollen estrategias de lectura que les permita identificar la estructura, ampliar su vocabulario, comprender lo que se comunica, establecer relaciones con su entorno, plantear hipótesis de causa y efecto, además de analizar hechos sociales, construir una opinión y tomar una posición frente a los mismos. 
Las configuraciones didácticas se enriquecen al hacer uso de textos en diferentes formatos. Estos textos permiten orientar las competencias para la lectura crítica de una manera intencionada. Ahora bien, el formato digital es más llamativo para los estudiantes porque es más dinámico e interactivo, por lo que se muestran más interesados y motivados a leer imágenes y videos que comprenden con mayor facilidad y con los que logran hacer una lectura crítica más consciente.

Es necesario resaltar que no hay una configuración didáctica única que favorezca la lectura crítica. Por el contrario, se comprueba que en la planeación estructurada del docente bajo una intencionalidad definida, se pueden favorecer procesos que logren despertar el interés y la motivación de los estudiantes en una variedad de actividades, donde ellos puedan apropiarse de los conocimientos de algunas de las competencias de un lector crítico, y así, ponerlos en juego para comprender lo que sucede a su alrededor, y también para establecer una interlocución con los diversos contextos que configuran la sociedad.

Es importante iniciar los procesos de formación en lectura crítica desde edades tempranas, haciendo uso de diferentes herramientas didácticas con el fin de enseñarles a los niños a cuestionarse sobre las ideas del autor, los géneros discursivos, las interpretaciones, tomar sus posturas y defenderlas con argumentos. Para ello, el maestro debe poseer un dominio teórico sobre las competencias a apropiar si se quiere formar lectores críticos, pues la lectura crítica necesita de una orientación precisa, acertada, planeada y estructurada para alcanzar expresiones críticas a través de posicionamientos y opiniones que son resultado de la interacción entre conocimientos, sentimientos, voluntades, las ideas y el contexto. De este modo, el docente juega un papel determinante en la promoción de la lectura crítica, pues debe propiciar espacios en los que los estudiantes encaren los diferentes textos con el objetivo de desentrañar, cuestionar y analizar en lo leído aquello que sucede a su alrededor.

En este contexto, es importante que el docente valore los aportes hechos por los estudiantes frente a la información leída, en los cuales logren resignificar su pensamiento a partir del diálogo establecido con el texto y las diferentes reflexiones que se suscitan en el grupo con el fin de evaluar el proceso de lectura crítica y el uso de sus diferentes competencias, de esta manera podrá fortalecer su quehacer pedagógico.

La formación de lectores críticos es necesaria y posible desde los primeros grados escolares porque los estudiantes, al apropiarse de algunas de las competencias de un lector crítico, se fortalecen en todo su ciclo escolar. Por ello, es importante que el docente considere las necesidades de sus estudiantes y el contexto en el cual están inmersos a la hora de elegir los contenidos temáticos y diseñar las estrategias pedagógicas que va a implementar en el aula, de ma- 
nera que ellos puedan ser partícipes en su proceso de aprendizaje, comprender, establecer relaciones y hacer uso de saberes para desenvolverse en el medio social de manera asertiva y comprometida con la transformación de su entorno.

\section{Agradecimientos}

Este artículo es resultado de la investigación titulada Prácticas de lectura crítica con estudiantes de los grados $2^{\circ}$ y $3^{\circ}$, una posibilidad de interlocución con su contexto a través de configuraciones didácticas, en el marco de la maestría en Educación de la Universidad de Medellín. La investigación fue orientada por la profesora Luz Adriana Restrepo Calderón.

\section{Referencias}

Álvarez-Álvarez, C. y Pascual-Díez, J. (2013). Estudio de caso sobre la formación de lectores críticos mediante textos literarios en la Educación Primaria. Ocnos, (10), 27-53. Recuperado de https:// revista.uclm.es/index.php/ocnos/article/view/ocnos_2013.10.02

Carantón, J. (2018). Apuntes sobre una educación conflictiva. Educación Sociedad y cultura, 110-121. Universidad Católica de Manizales, Manizales.

Camps, A. y Ribas, T. (2003). La evaluación del aprendizaje escolar de la composición escrita en situación escolar. Memoria de investigación - Concurso nacional de proyectos de investigación educativa. Ministerio de educación, cultura y deporte. Madrid, España: Secretaría general técnica, centro de publicaciones.

Cardona, P. y Londoño, D. (2017). El sentido de la lectura crítica en contexto. Katharsis, (22), 375-401. Recuperado de http://revistas.iue.edu.co/index.php/katharsis/article/view/835

Cassany, D. (2003). Aproximaciones a la lectura crítica: teoría, ejemplos y reflexiones. Tarbiya. Revista de investigación e innovación educativa, 32, 113-132.

Cassany, D. (2005). Expresión escrita en L2/ELE. Madrid, España: Arco Libros.

Cassany, D, García Del Toro, A. (2001) Recetas para escribir. Madrid Editorial Plaza Mayor.

Cassany, D. (2007). Ensenar lengua/ Learning the Language. Madrid, España: Editorial Grao.

De Zubiría, J. (2015). ¿Democracia sin lectura crítica? Revista Semana. Recuperado de https://www. semana.com/educacion/articulo/lectura-critica-para-la-democracia/447801-3

Delgado Uriarte, C. (2013). La lectura crítica, una herramienta de formación del pensamiento crítico en la universidad. Redem. Recuperado de https://www.redem.org/la-lectura-critica-unaherramienta-de-formacion-del-pensamiento-critico-en-la-universidad/

Freire, P. (1986). Hacia una Pedagogía de la Pregunta: conversaciones con Antonio Faúndez. Buenos Aires, Argentina: Ediciones La Aurora.

Jurado, F. (2008). La formación de lectores críticos desde el aula. OEI Revista Iberoamericana de educación, (46), 89-105. Recuperado de https://rieoei.org/historico/documentos/rie46a05.htm

Lerner, D. (2002). La autonomía del lector. Projeto. Revista de Educacão, $\mathrm{N}^{\circ} 4, \mathrm{~N}^{\circ} 6$, maio, Editora Projeto, Porto Alegre, Brasil. 
Litwin, E. (1997). Las configuraciones Didácticas: Una Nueva Agenda para la Enseñanza Superior. Buenos Aires, Argentina: Paidós.

Luke, A. (2004). Two takes on the critical. En B. Norton y K. Toohey (Eds.), Critical pedagogies and language learning (pp.21-29). Cambridge: Cambridge University Press.

Medina Moya, L. (2011). El conocimiento didáctico del contenido. un estudio de casos múltiple con profesores universitarios de distintas áreas de conocimiento. III Congreso Internacional de Nuevas Tendencias en la Formación Permanente del Profesorado, Barcelona, España.

Ministerio de Educación Nacional. (1998). Lineamientos Curriculares de Lengua Castellana. Bogotá, Colombia: Magisterio.

Ministerio de Educación Nacional. (2006). Estándares Básicos en Competencias del lenguaje. Bogotá, Colombia: Magisterio.

Ministerio de Educación Nacional. (2016). Guía de orientación. Módulo de Lectura crítica Saber Pro 2016-2. Bogotá, Colombia: Icfes.

Ministerio de Educación Nacional. (2015). Derechos Básicos de Aprendizaje. Bogotá, Colombia: Magisterio.

Murcia, L. (2012). Hacia la formación de lectores críticos: Un estudio de caso de clases de historia y literatura. (Tesis de Maestría) Instituto de Investigación en Educación, Universidad Nacional, Bogotá, Colombia. Recuperado de http://www.bdigital.unal.edu.co/9002/1/Linamireyamurciadiaz.2012.pdf

Perkins, D. (1999). Qué es la comprensión. En Stone, M. (Comp.). La enseñanza para la comprensión entre la investigación y la práctica. Buenos Aires, Argentina: Paidós.

Serrano, S., Madrid, A. (2007). Competencias de lectura crítica. una propuesta para la reflexión... Acción pedagógica, (16), 58-68.

Zañartu, L. (2003). Aprendizaje colaborativo: Una nueva forma de diálogo interpersonal en red. Revista digital de Educación y nuevas Tecnologías. Recuperado de https://tic.sepdf.gob.mx/micrositio/ micrositio2/archivos/AprendizajeColaborativo.pdf

Zuleta, O. (2005). La pedagogía de la pregunta. Una contribución para el aprendizaje. Educere, 9(28), 115-119. Zuluaga T., C. (2016). Formación de lectoras críticas desde la literatura hacia otros sistemas simbólicos Repositorio Universidad de Antioquia. http://hdl.handle.net/123456789/2383 